EXPERIMENTS AND DETECTORS

FOR

HIGH ENERGY HEAVY ION COLLIDERS

T. Ludlam

Brookhaven National Laboratory

October 22, 1985 


\section{EXPERIMENTS AND DETECTORS FOR HIGH ENERGY HEAVY ION COLLIDERS}

T. Ludlam

Brookhaven National Laboratory, Upton, New York 11973

Presented at

CONFERENCE ON INSTRUMENTATION FOR HEAVT ION NUCLEAR RESEARCH

Oak Ridge National Laboratory, Oak Ridge, Tennessee 37831 October 22-24, 1984

The submitted manuscript has been authored under contract DE-AC02-76CH00016 with the U.S. Department of Energy. Accordingly, the U.S. Government retains a nonexclusive, royalty-free license to publish or reproduce the published form of this contribution, or allow others to do so, for U.S. Government purposes. 
CHAPTER I

EXPERIMENTS AND DETECTORS FOR HIGH ENERGY HEAVY ION COLLIDERS

T. LUDLAM

Physics Department

Brookhaven National Laboratory

Upton, New York

1. INTRODUCTION: The Nature of the Experiments

In the previous talk, by Lee Schroeder, 1 you have seen an overview of the detector considerations for future experiments with high energy nuclear beams. By and large, these experiments are shaped by the expectations for new phenomena to be realized when nuclear matter is brought to extreme states of temperature and density. Lee has concentrated on the fixed-target experiments which are being prepared for the relatively near future at Brookhaven and CERN: experiments at collision energies which are expected to approach the limit of nuclear transparency and thus produce high energy density through compression and heating of baryon-rich nuclear matter.

In the present talk my focus will be on the problems and possibilities for experiments at the highest collision energies achievable in man-made accelerators; i.e. colliding beams of heavy nuclei at c.m. energies $>100 \mathrm{GeV} / \mathrm{amu}$, well beyond the threshold of nuclear transparency. Here the 


\section{T. LIDLAM}

final state consists of two hot, dense, baryon-rich fireballs flying away from each other at large rapidity (the fragmentation regions), and thermally-produced particles with near-zero net baryon number populating the central rapidity range. The matter produced at central rapidity (the lab frame for a collider) may reach extremely high temperatures and energy densities, and it is here that one expects to produce thermodynamic conditions similar to those which existed when the early universe condensed from a plasma of quarks and gluons to a gas of hadrons.

Figure $I$ shows the layout of the Brookhaven accelerator complex, including the partially-completed ISABELLE collid- ing beams accelerator which is now being proposed. as a dedicated Relativistic Heavy Ion Collider (RHIC). ${ }^{2}$ For the sake of specificity the discussion here will assume a collider facility with the design parameters of RHIC: beam masses and energies up to gold $(A \approx 200)$ at $100 \mathrm{GeV} / \mathrm{amu}$ with luminosity L) $10^{26} \mathrm{~cm}^{-2} \mathrm{sec}^{-1}$.

We are familiar with many. examples of large, expensive and extraordinarily complex detection systens for studying collisions of elementary particles in $e^{+} e^{-}, p p$ and $\overline{p p}$ colliders. Detectors at a heavy ion collider, while they may also be complex and expensive, are likely to be very different in their design and function from their counterparts in high energy physics experiments. Machines such as IEP, the pp colliders, and the Superconducting Super Collider are undertaken to explore the realm of hard scattering processes to resolve phenomena at the shortest possible interaction distances. This leads to experiments detecting leptons and jets at high transverse momenta, which are rare events, and little or no concern for sensitivity to the "soft" particles which are the typical reaction products. 


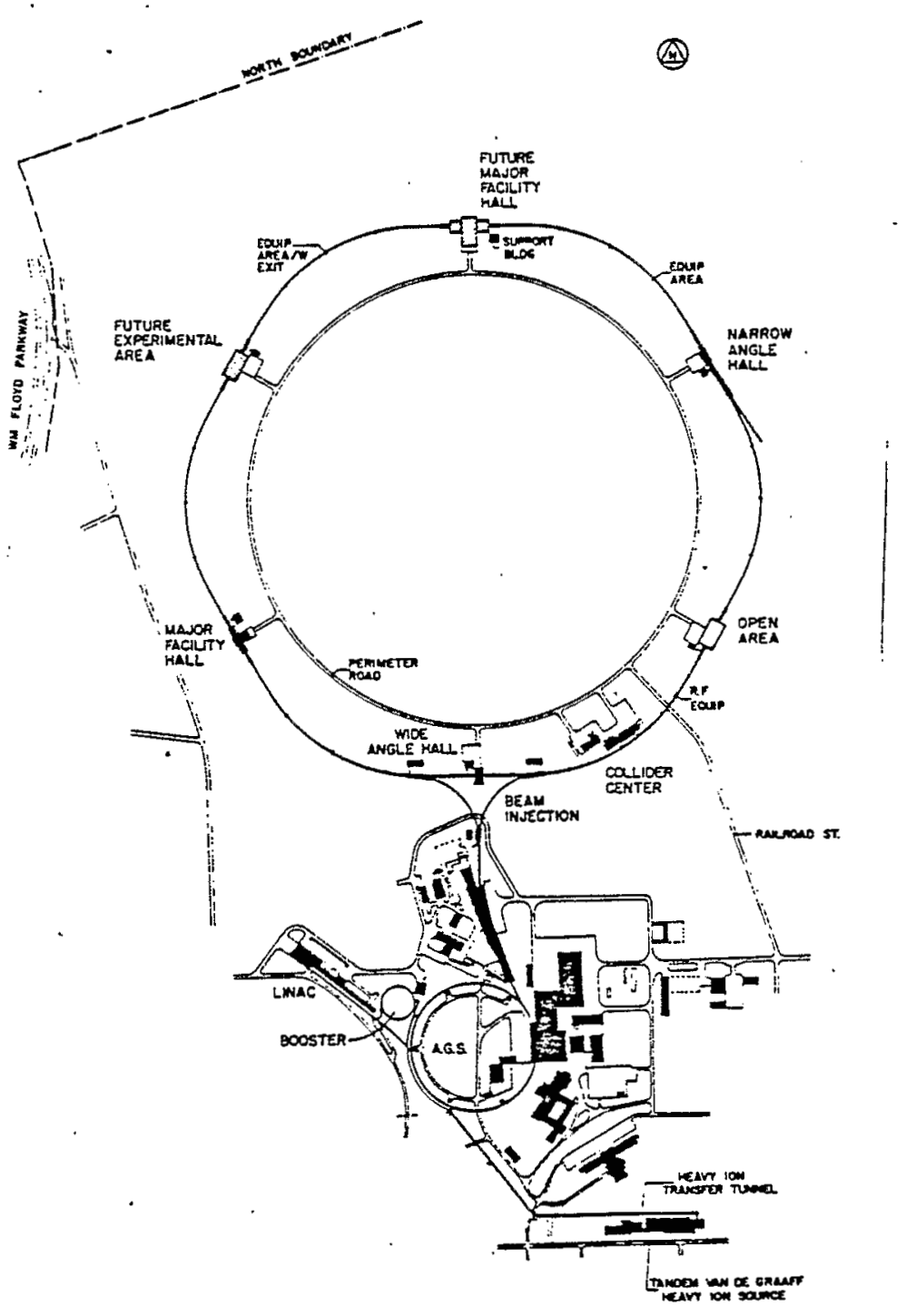

Fig. 1. Site map of the Brookhaven accelerator complex, showing the $30 \mathrm{GeV}$ Alternating Gradient Synchrotron (AGS) proton accelerator with heavy ion injection from the Tandem Van de Graaff facility. The AGS would serve as injector to the (unfinished) colliding beams facility. 
T. LUDLAM

TABIE I. Experimental signals for new states of matter.

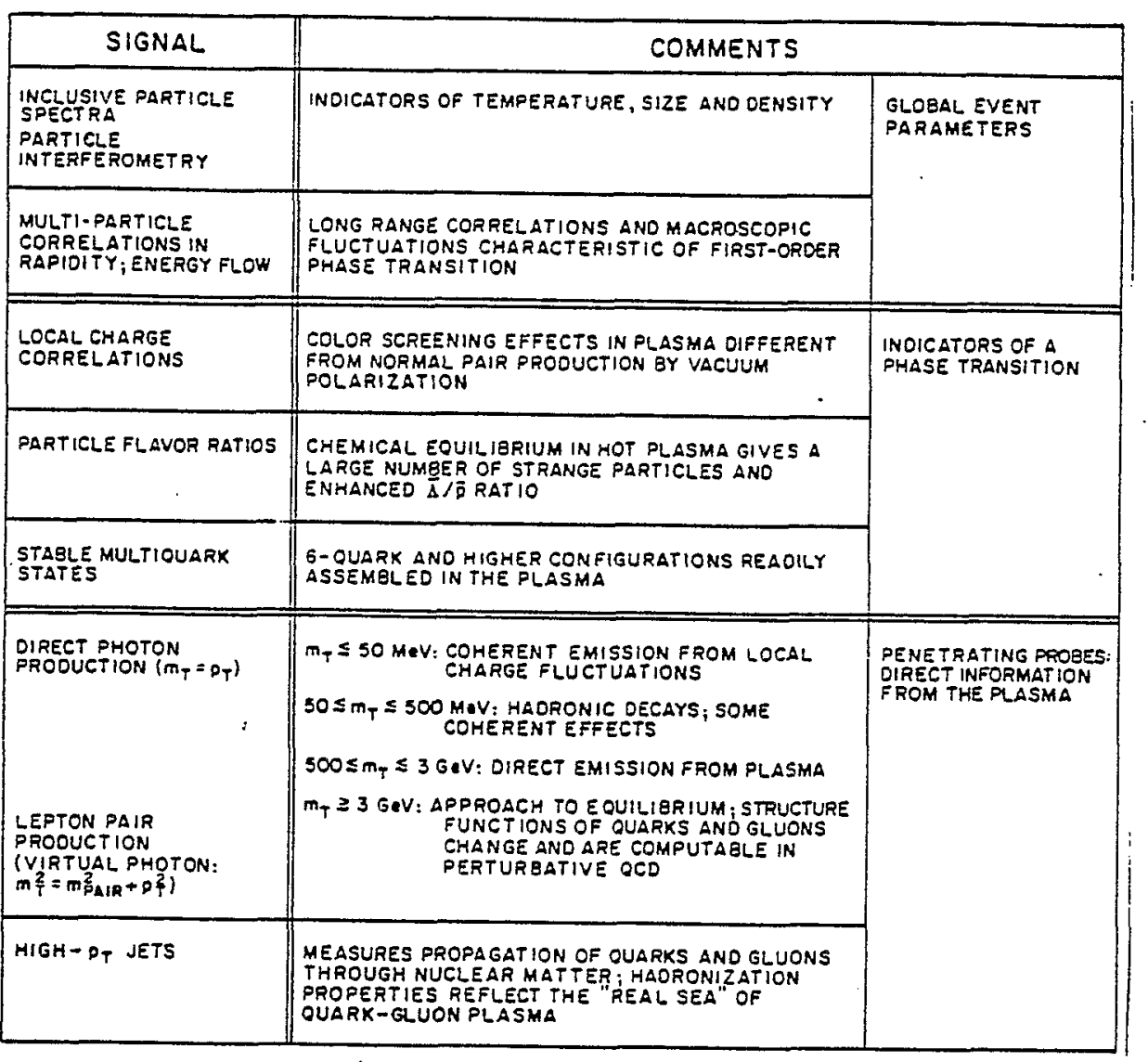


EXPERIMENTS AND DETECTORS FOR HIGH ENERGY HEAVY ION COLLIDERS

By contrast, the quark-matter physics of high energy nuclear. collistions emphasizes the long distance aspects of QCD, and the signals of interest involve particles whose momenta are characteristic of the equilibrium thermodynamic conditions reached in the collision. This means that most experiments will be designed to look for signals carried by relatively soft particles. Table I summarizes the kinds of signais which are often discussed. ${ }^{3}$ They emphasize measurement of detailed global patterns of energy flow (in which the importance of individual particle measurements is suppressed); the relative rate of heavy flavor production (strangeness and charm); short-range correlations among small numbers of particles, including resonance masses and particle interferometry (the latter is a completely new form of measurement made possible by the high multiplicity and large spatial extent of interesting events - these measurements require precise measurement of individual tracks, possibly over a limited solid angle); and the detection and reconstruction of relatively rare froms of particle emission which are thought to serve as "penetraring probes" of the initial stages of the nuclear collision (virtual photons, seen as lepton pairs, or hard-scattered quarks and gluons seen as jets). In general these types of measurements must be made on an event-by-event basis, with some kind of trigger selection on small impact parameter and large energy depostion. The final states thus selected will be very different from those encountered in high energy physics experiments: the particle multiplicity may range into the thousands. An educated guess at the average rapidity distribution of charged particles for three different collider energies is shown in Figure 2. Figure 3 relates the rapidity spectrum to intervals of laboratory angle in a possible 
T. LUDLAM

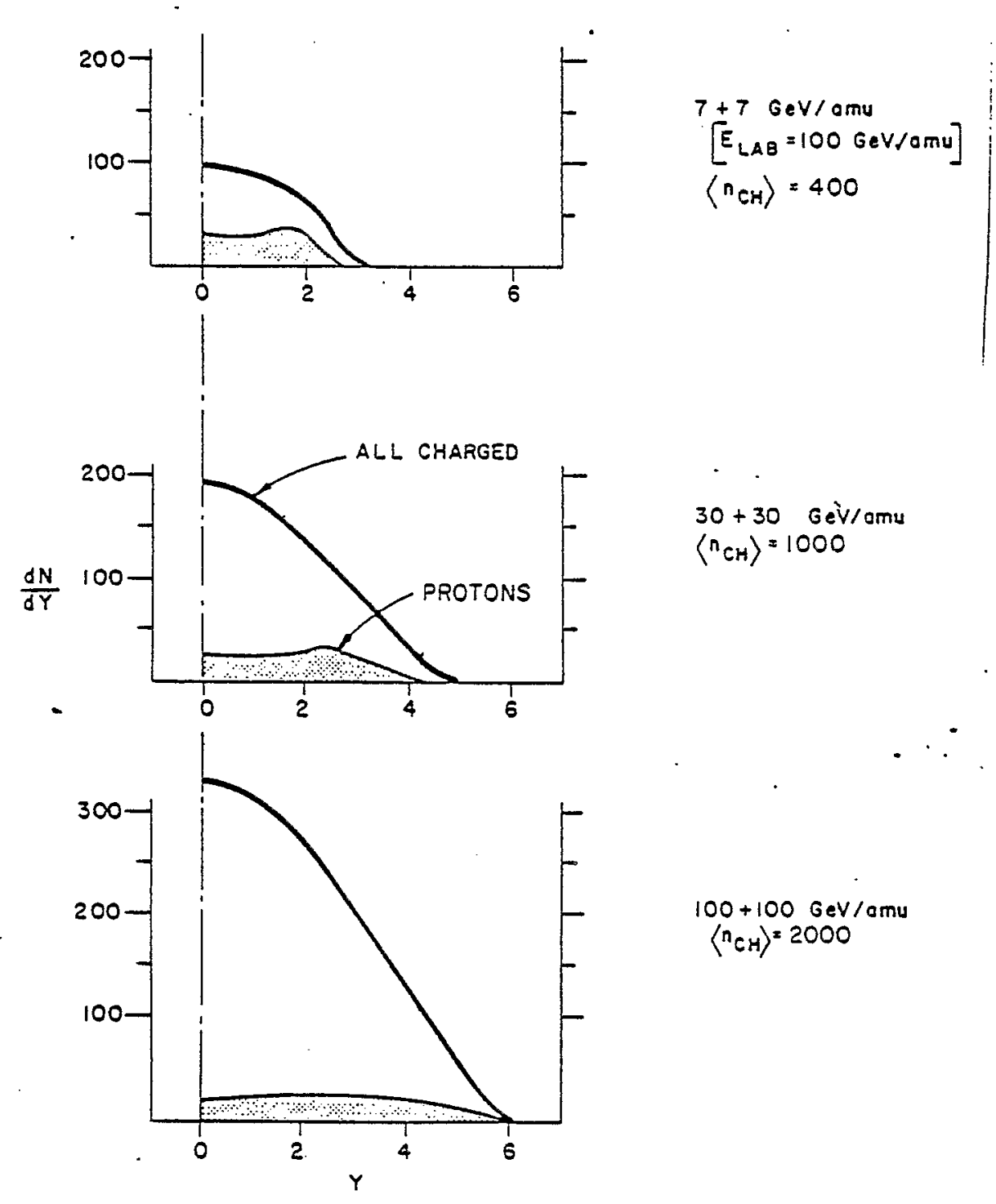

Fig. 2. Illustrating the expected charged particle spectra for Au + Au collisions at various collider energies. The shaded areas are the net proton densities extrapolated from the stopping power measurements in proton-nucleus collisions. $\left\langle\mathrm{n}_{\mathrm{CH}}\right\rangle$ is the mean multiplicity of charged particles. 
EXPERIMENTS AND DETECTORS FOR HIGH ENERGY HEAVY ION COLLIDERS

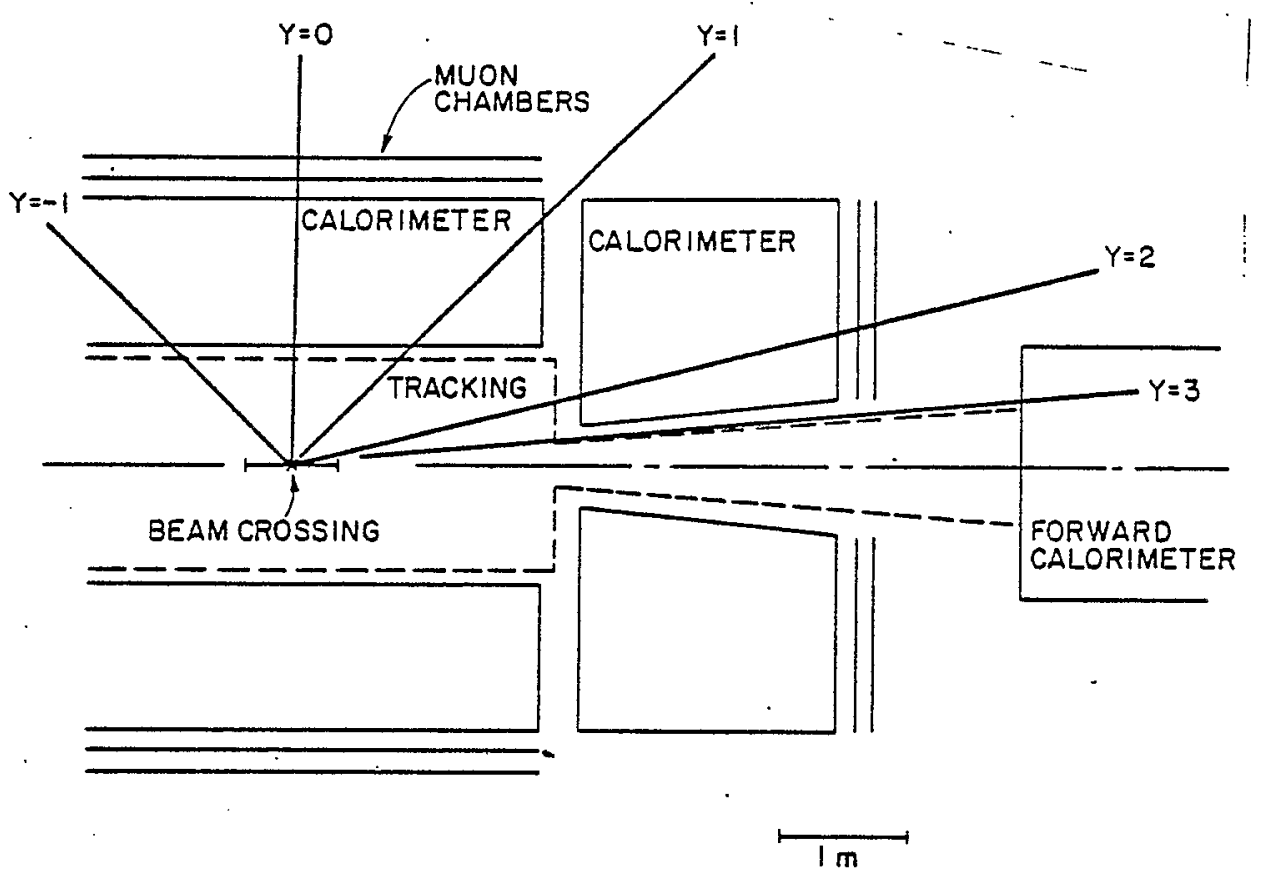

Fig. 3. Schematic layout showing the geometry and kinematics of a detector system for colliding beams experiments. The angular intervals corresponding to various rapidity $(y)$ values are indicated. A full solid-angle detector would have the same coverage on either side of $\mathrm{y}=0$; this illustration shows coverage of the central region and one of the fragmentation regions. 


\section{T. LUDIAM}

layout of detectors.

In the following sections. I will examine some specific detection problems for these experiments. The required detection technology for tracking, calorimetry, particle identification and fast trigger decisions has a great deal in common with components of high energy physics experiments. For general discussions of the various detector types and their elementary properties some suggested sources are given in Refs. 3-5.

2. THE PROBLEM OF TRACKING

The problem of tracking in these experiments is one of dealing with extraordinarily high multiplicities of particles: upwards of 1000 charged tracks in the central rapidity region (Fig. 2), corresponding to $\sim 200$ per steradian of solid angle. In point of fact; this density of tracks is not greater than those encountered in present experiments looking at high-p $\mathrm{p}_{\mathrm{T}}$ jets. The measurement of local track denstties of this magnitude is not beyond the capability of present detector technology. The difficulcy, of course, is that in nuclear collision events the detectors will see such densities uniformly over all angles.

It may be asked, with so many final-state particles per event, whether it is necessary to resolve individual tracks in order to determine the salient event parameters. Indeed it is true that many of the global properties of events can be obtained through local averages over many particles, e.g. with calorimeter measurements, in ways that would not be possible, because of large fluctuations, in elementary particle collisions.

Nonetheless, we have seen that there are cases where it is desirable to resolve single particles in high multi- 
EXPERIMENTS AND DETECTORS FOR HIGH ENERGY HEAVY ION COLIIDERS.

plicity nuclear collisions: the detection of strange particles and leptons, and the measurement of correlation functions for particle interferometry are sose examples. It may be acceptable to make such measurements over a limited solid angle, sampling a small fraction of the total final-state particle count. In this case today's advanced, three-dimensional "imaging detectors" are up to the job. For example, the time projection chamber (TPC) being built for the ALEPH experiment at LEP is designed to handle track densities up to $\sim 1000 /$ steradian in jets which are localized to .01 steradian. 6 Other types of wire chanbers, less expensive and with better rate capability than the TPC, are being refined to the point where they may be considered for such applications. Thus, the two-track resolution in the drift direction in the conventional wire chanber is typically on the order of $5 \mathrm{~mm}$. Improvements in electronics, electrode geometry, and gas chemistry have reduced this by an order of magnitude in practical prototypes that are now being built.'

If it is necessary to cover large solid angles, recording hundreds of tracks in a single event, the difficulty lies not in the technical limitations of the detectors themselves, but in the data acquisition and processing. The system, consisting of detector and track reconstruction algorithn, must be designed so that the processing time remains approximately linear with the number of tracks up to very high track counts. The intrinsic limits have not been. explored in real experiments, although studies of a particular case indicate that the level of 1000 tracks per event can be reached with presently understood techniques.? 


\section{T. LUDIAM}

\section{LEPTON MEASUREMENTS}

Effective measurement of low-mass electron pairs in the high multiplicity environment of nucleus-nucleus interactions is widely recognized as an important and formidable challenge for the design of experiments. Detailed calculations of detector response and background rates have been carried out for specific cases of experiments planned for fixed target operation.

The instrumentation must be capable of distinguishing electrons from a high flux of background hadrons: electron to hadron rejection at a level $=10^{5}$ is required. In the collider case this might be accomplished in a specialized detector of relatively small aperture of $90^{\circ}$ in the c.m. with the tracks most favorably disposed in laboratory angle. For efficient detection of low-mass pairs a two-arm detector would be required (the arms back-to-back). Successive, independent Cerenkov measurements would provide good hadron rejection. The Cerenkov counters have to be well segmented. If ring-imaging Cerenkov detectors ${ }^{12}$ are developed to their apparent potential, one has a very nice solution for this configuration: a multitrack detector with good position resolution which can be made effectively blind to the multitude of background hadronic tracks.

These technical requirements for electron/hadron discrimination notwithstanding, the most severe fundamental limitation to the study of electron pairs in the mass and $\mathrm{p}_{T}$ range $\lesssim I \mathrm{GeV}$ is the level of background, electrons from secondary processes. Given the charged particle densities indicated in Fig. 2, we can expect in excess of $100 \pi^{\circ}$ particles per unit of rapidity in the central region. Thus on the average there will be $\approx 1 e^{+} e^{-}$pair per unit of 
EXPERIMENTS AND DETECTORS FOR HIGH ENERGY HEAVY ION COLLIDERS rapidity from the decay $\pi^{\circ}+e^{+} e^{-} \gamma$, and $>200$ photons from $\pi^{\circ} \rightarrow \gamma \gamma$. If the detectors present only $2 \%$ of a radiation length of material thickness to induce pair conversions, the net result is >10 electrons per unit rapidity in each event. This background can only be eliminated by correctly reconstructing all electrons and removing pairs with mass > $100 \mathrm{MeV}$. Design studies with detector systems optimized for this task (see Refs. 9, 10, 11) have rather consistently concluded that the limiting sensitivity for $e^{+} e^{-}$pairs in the mass range $.5-1 \mathrm{GeV}$ is

$$
\frac{n\left(e^{+} e^{-}\right)}{n\left(\pi^{5}\right)}>10^{-5}
$$

Specialized techniques for electron identification are not so easily incorporated in a large solid angle, general purpose detector such as that shown in Fig. 3. In the central rapidity region such a detector may achieve some e/ $\pi$ rejection through $\mathrm{dE} / \mathrm{dx}$ measurements, if sensitivity is sufficiently good to work in the relativistic rise regime. This is a severe requirement for a detector which must also achieve efficlent tracking at high multiplicities and remain relatively compact. Some additional hadron rejection may be gained with fine-grain segmentation in the first layers $(\approx 20$ rad.len.) of the calorimeter. Very soft hadrons ( $B Y \lesssim 2$ ) may be rejected by time-of-flight, even in a compact geometry with $\approx 1$ meter flight path, if the timing accuracy promised by Pestov, cointers can be realized in a practical way. ${ }^{13}$ In the forward directions, where the laboratory momenta are high compact transition radiation detectors may be used for electron identification with good segmentation and hadron rejection as illustrated in Fig. 4. All of these techniques require efficient and precise tracking and momentum measurement. 
T. LUDIAM

Muons are identified by their ability to penetrate a thick absorber, and for high momentum muons this provides a straightforward means of reducing the high multiplicity hadronic background. Muons of momentum $P \mu>5 \mathrm{GeV} / \mathrm{c}$ are required for effective filtering of hadrons.' Thus, in the collider geometry one may not be able to efficiently measure low-mass, low $P_{T}$ muon pairs in the rapidity range near $y=0$. The main region of interest in the spectrum of transverse mass of muon pairs is (see Table. I) $M_{T} \sim 1-10 \mathrm{GeV}$. As with elections, the direct muon signal is threatened by a potentially disastrous background from secondary processes originating with the enormous number of final-state hadrons. In this case the problem is with muons from pion decay. An experiment dedicated to the measurement of $\mu^{+} \mu^{-}$pairs might take the approach of the Mark $\mathrm{J}$ experiment at PETRA, 15 shown in Fig. 5. Here an active absorber (calorimeter) immediately surrounds the interaction region, providing energy flow information and removing hadrons before they decay, followed by layers of magnetized iron interleaved with drift chambers to measure the trajectories of muons. For the nuclear beams case it will be critical to achieve rapid absorption of the mesons: An effective flight path of even $10 \mathrm{~cm}$ before absorption, a distance comparable to hadronic interaction lengths in dense materials, would still allow a finite probability for several fake pairs. Thus a properly designed experiment may require special beam pipes and carefully crafted compromises in the measurements of energy flow in the initial absorber in order to reduce the background to a manageable level. Such compromises will also affect the accessibility of the low end of the interesting mass range near $y=0$. 
EXPERIMENTS AND DETECTORS FOR HIGH ENERGY HEAVY ION COLLIDERS

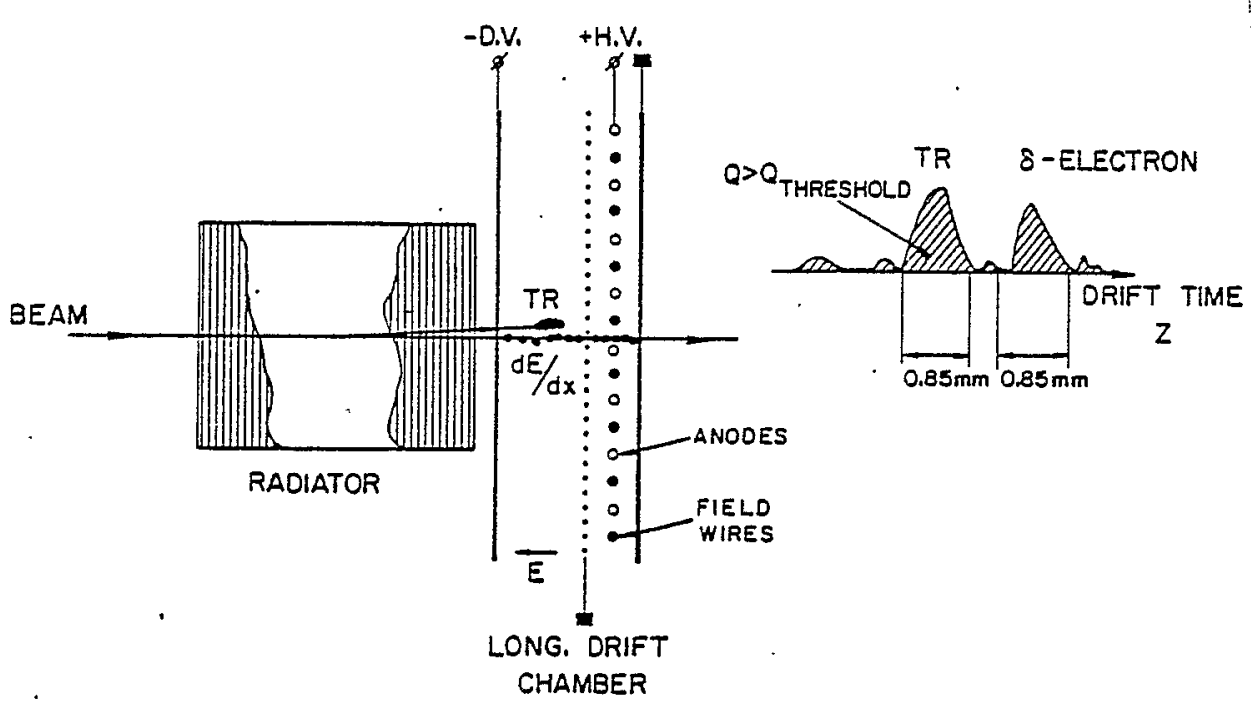

Fig. 4. Diagram showing the principle of transition radiation detection by "cluster counting" technique. The drift chamber photon detector and its high speed readout electronics are designed to resolve Individual ionization electrons in the wire chamber. In this way the $d E / d x$ signals from charged tracks (small clusters) can be distinguished from the signals due to absorption of x-ray quanta (large clusters), and the sensitivity to the TR signal can be significantly enhanced. (see Ref. 14). 
T. IUDLAM

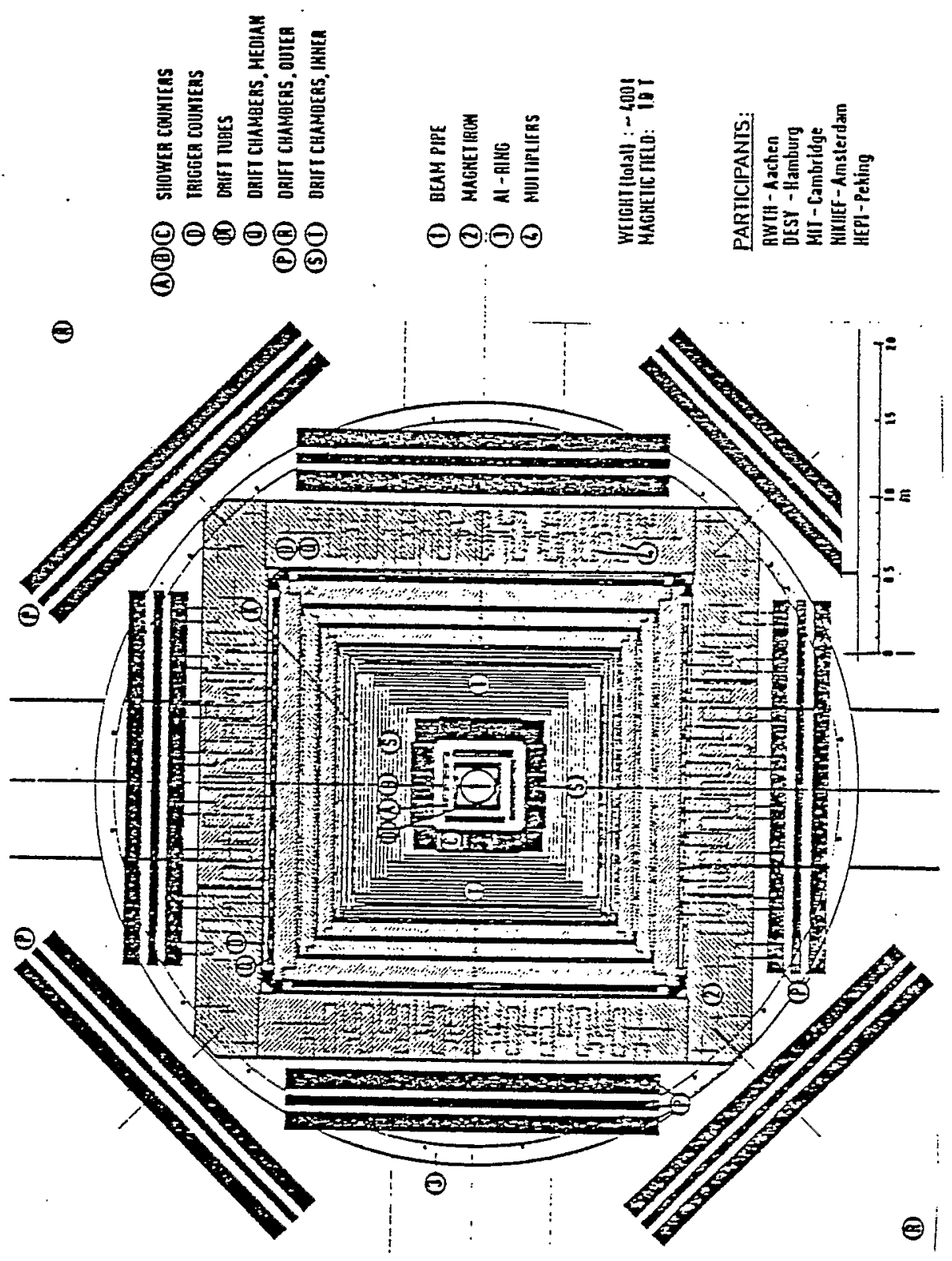

Fig. 5 View of Mark $\mathrm{J}$ detector for $\mu+\mu^{-}$measurements in colliding electron beams at PETRA. The beam direction is perpendicular to the plane of the drawing (Ref.15). 
EXPERIMENTS AND DETECTORS FOR HIGH ENERGY HEAVY ION COLIIDERS

\section{CALORIMETERS}

Calorimeters measure the energy, position and direction of particles through total absorption of the incident. energy in a dense material that has been laced with a matrix of active readout elements. The technology of these detectors has recently been brought to a high level of sophistication for many types of application in colliding beam experiments. 5 For nuclear beams experiments these devices will provide a powerful means of averaging over the single particle behavior to give precise patterns of energy flow, with signals developed on a time scale ( $\lesssim 100$ nanosec) which is useful for very fast trigger decisions. Calorimeters provide the only practical means of measuring the energy carried by neutral particles.

The limiting energy resolution of a calorimeter is determined by fluctuations intrinsic to the mechanism of shower development. The underlying phenomena are statistical processes whose effects grow in magnitude as $E^{1 / 2}$, where $E$ is the deposited energy. Hence the limiting accuracy, expressed as a fraction of the total energy, improves with increasing energy deposit as $\mathrm{E}^{-1 / 2}$. For sampling calorimeters, typical values for the fractional energy 5 resolution $(\sigma / E)$ and transverse shower size $\left(\mathrm{X}_{\mathrm{T}}\right)$ are:

Electromagnetic Showers (electrons and photons):

$$
\frac{\sigma(E)}{E}=\frac{12}{\sqrt{E}(\mathrm{GeV})} ; \mathrm{X}_{\mathrm{T}}=1 \mathrm{~cm}
$$

Hadronic Showers:

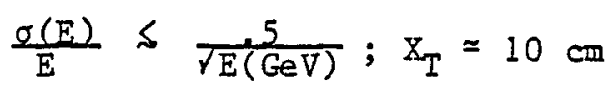




\section{T. LUDLAM}

The value of $\mathrm{x}_{\mathrm{T}}$ gives the minimum physical size of individual read-out elements. The degree of angular segmentation is then determined by the distance of the calorimeter face from the interaction point. Figure 6 shows a calorimeter array consisting of four walls surrounding the intersection point of the colliding beans at the CERN ISR. 16 The calorimeter stacks, which here consist of interleaved layers of uranium, copper and plastic scintillator, are 1.2 meters thick. Each wall is subdivided into a checkerboard array of $20 \mathrm{~cm} \times 20 \mathrm{~cm}$ "towers" which are read out individually. The tower size is determined by the dimensions of hadronic showers. For detection of electrons and photons the segmentation can be much finer: Fig. 7 shows, from the same experiment, an array of $4 \times 4 \mathrm{~cm}^{2}$ sodium iodide crystals which is placed on the inside face of one of the calorimeter walls.

In contrast to our problems with tracking, the performance of a calorimeter system such. as "this should be excellent for the high multiplicity nuclear beams case. In a collider with the parameters of RHIC, for instance, where Gold beams interact at $100+100 \mathrm{GeV} / \mathrm{amu}$, the $~ 3000$ finalstate particles in a head-on collision carry away as much as $40 \mathrm{TeV}$ of energy.

A calorimeter system covering the full solid angle and subdivided into 1000 cells would have enough energy deposited in each cell for an energy measurement at the level of 10\%. The angular distribution of the energy flow in each event is thus determined with extraordinary precision. The calorimeter need not be too deep, since the energy is carried by a large number of relatively low energy particles - an important difference from the design critteria for current high energy physics experiments. Also, a $4 \pi$ calorimeter for 
EXPERIMENTS AND DETECTORS FOR HIGH ENERGY HEAVY ION COLLIDERS

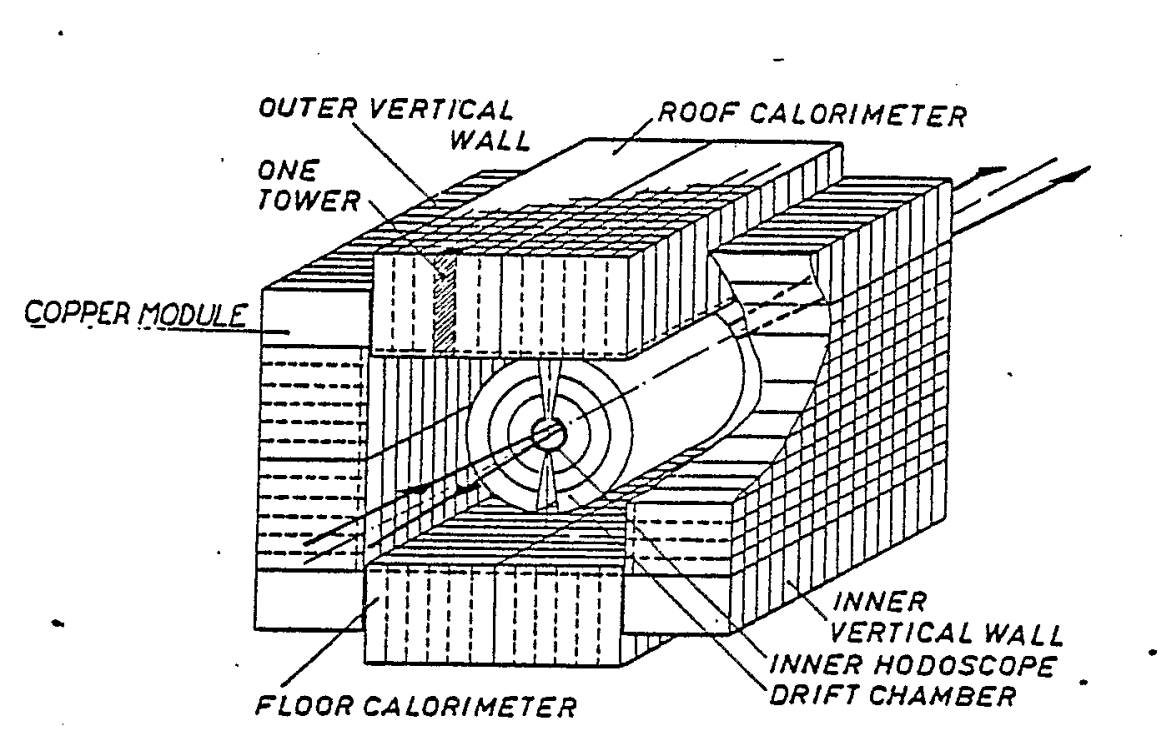

Fig. 6. The Detector Array of the Axial Field Spectrometer (CERN experiment R807/808) surrounding the ISR colliding beams (Ref. 16). 
T. IUDLAM

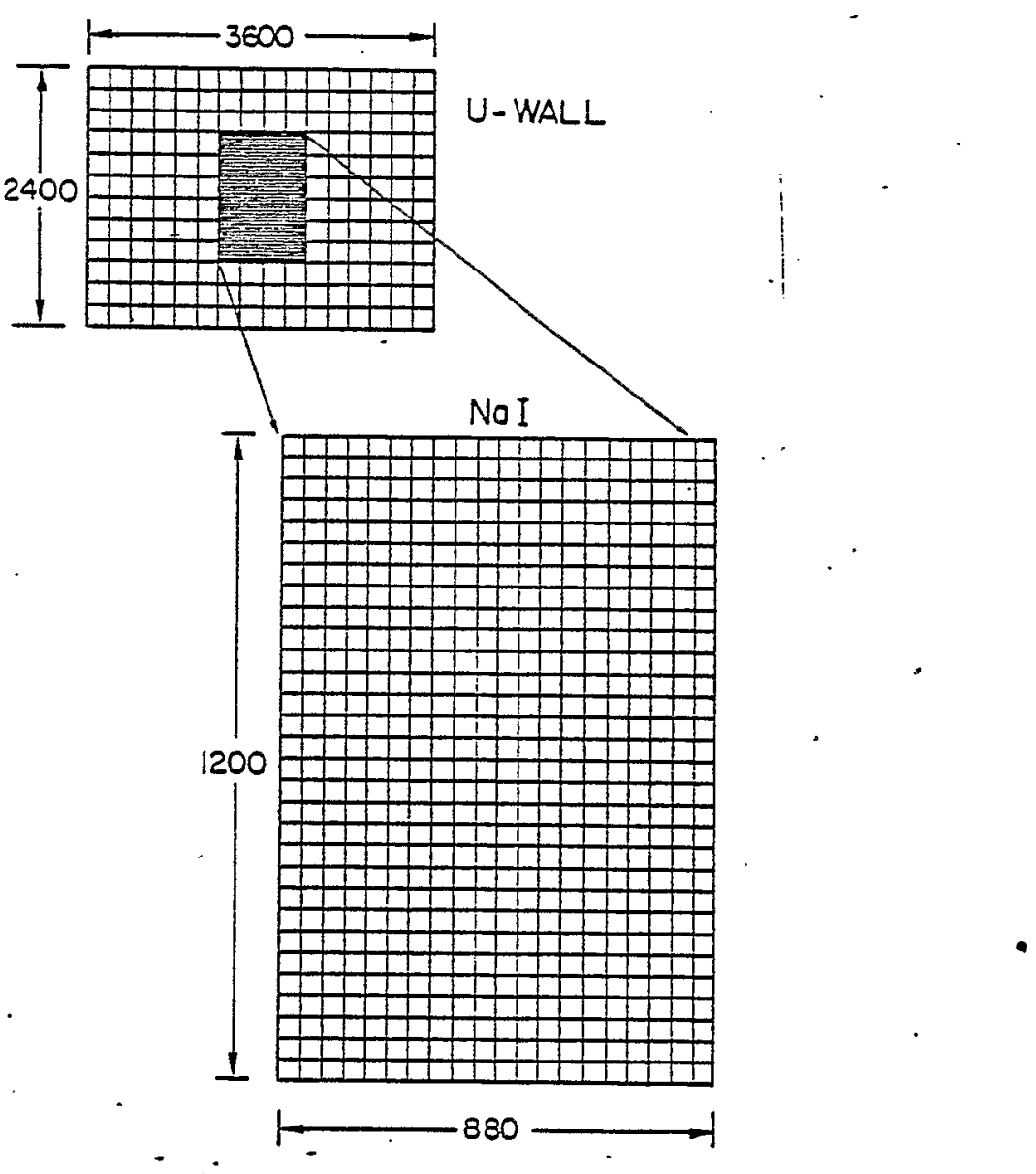

Fig. 7. An array of 600 sodium iodide crystals, each $4 \times 4 \mathrm{~cm}^{2}$, covering an area $\sim 1$ square meter on the face of a uranium calorimeter wall of the Axial Field Spectrometer. 
EXPERIMENTS AND DETECTORS FOR HIGH ENERGY HEAVY ION COLLIDERS nuclear beams experiments need not be rigorously "hermetic": The physics considerations which guide event selection do not hinge on cases where a large fraction of the total incident energy may be carried away by a single particle, and so the design can tolerate some cracks and other gaps in the coverage if necessary.

These considerations lead us to a detector concept unique to the physics of a high energy heavy ion collider: ${ }^{17}$ a nearly - $4 \pi$ calorimeter facility in wich a number of small apertures are provided for special-purpose spectrometers. Events are selected according to precisely measured patterns of energy flow, while the several instrumented "ports" provide detailed measurements on individual particles in spectrometers whose apertures are small enough so that the tracking problem is manageable.

This work was supported by the U.S. Department of Energy under Contract DE-ACO2-76-H00016. 
T. LUDLAM

\section{REFERENCES}

1. L. SCHROEDER, "Report on the LBL Workshop on Detectors for Relativistic Nuclear Collisions," these proceedings.

2. Brookhaven Proposal for a Relativistic Heavy Ion Collider, BNL 51801 (1984); see also: T. LUDLAM "RHIC and Quark Matter: A Proposed Heavy Ion Collider at Brookhaven National Laboratory", BNL 35237, to appear in Proc. of the Fourth International Conference on UltraRelativistic Nucleus-Nucleus Collisions, Helsinki, June 1984.

3. Phys. Today, October 1978, A special issue on "Detecting Particles."

4. C. W. FABJAN and H. G. FISCHER, "Particle Detectors", Rep. Prog. Phys., 43, 1003 (1980).

5. C. W. FABJAN and T. LUDLAM, "Calorimetry in High Energy Physics", Ann. Rev. Mucl. Part. Sci., 32, 335 (1982).

6. ALEPH collaboration, CERN proposal, LEPC/82-3II (1982).

7. W. WILLIS, Nucl. Phys. A418, 425c (1984).

8. G. CHARPAK and F. SAULI, Ann. Reỵ. Nucl. and Part. Sci. 39, 285 (1984).

9. D. HENDRIE, "Dilepton Spectrometer Based on the Lampshade Magnet", Nucl. Phys. A418, 647c (1984).

10. H. GORDON et al., "Proposal for CERN Experiment NA34", CERN-SPSC/83-51 (1983).

11. H. SPECHT, "Detection of Photons and Leptons in High Energy Nuclear Collisions," These Proceedings.

12. B. BARRELET et al., Nucl. Instr. Meth. 200, 219 (1982).

13. Y. PESTOV and W. ATWOOD, Proc. IntI. Conf. on Instrumentation for Colliding Beam Physics, SLAC, 1982. 
EXPERIMENTS AND DETECTORS FOR HIGH ENERGY HEAVY ION COLLIDERS

REFERENCES (cont'd)

14. T. LUDLAM et al., Nuc1. Instr. Meth. 180, 143 (1981);

C. W. FABJAN et al., Nucl. Instr. Meth. 216, 105

(1983).

15. Mark J. Collaboration, Phys. Reports 63, 337 (1980).

16. T. AKESSON et. al.,.z Phys. C-25, 13 (1984).

17. W. WILLIS, private communication. 